\title{
Strut Support with Tricortical Iliac Allografts in Unstable Proximal Humerus Fractures: Surgical Indication and New Definition of Poor Medial Column Support
}

\author{
Seung-Jin Lee, Yoon-Suk Hyun ${ }^{\varpi}$, Seung-Ha Baek \\ Department of Orthopadic Surgery, Kangdong Sacred Heart Hospital, Seoul, Korea
}

Background: The execution of fibular allograft augmentation in unstable proximal humerus fractures (PHFs) was technically demanding. In this study, the authors evaluated the clinical and radiographic outcomes after tricortical iliac allograft (TIA) augmentation in PHFs. Methods: We retrospectively assessed 38 PHF patients treated with locking-plate fixation and TIA augmentation. Insertion of a TIA was indicated when an unstable PHF showed a large cavitary defect and poor medial column support after open reduction, regardless of the presence of medial cortical comminution in preoperative images. Radiographic imaging parameters (humeral head height, $\mathrm{HHH}$; humeral neck-shaft angle, HNSA; head mediolateral offset, HMLO; and status of the union), Constant score, and range of motion were evaluated. Patients were grouped according to whether the medial column support after open reduction was poor or not (groups A and B, respectively); clinical outcomes were compared for all parameters.

Results: All fractures healed radiologically (average duration to complete union, 5.8 months). At final evaluation, the average Constant score was 73 points and the mean active forward flexion was $148^{\circ}$. Based on the Paavolainen assessment method, 33 patients had good results and 5 patients showed fair results. The mean loss of reduction was $1.32 \mathrm{~mm}$ in $\mathrm{HHH}$ and $5.02 \%$ in HMLO. None of the parameters evaluated showed a statistically significant difference between the two groups (poor and not poor medial column support).

Conclusions: In unstable PHFs, TIA augmentation can provide good clinical and radiological results when there are poor medial column support and a large cavitary defect after open reduction.

(Clin Shoulder Ellbow 2019;22(1):29-36)

Key Words: Tricortical iliac allograft; Allograft augmentation; Proximal humerus fracture

\section{Introduction}

Proximal humerus fractures (PHFs) commonly occur in elderly patients with decreased bone mineral density. Local osteopenia and fracture comminution can compromise the stability of internal fixation techniques and may lead to hardware failure, fracture re-displacement, and a poor outcome."

Many methods of fixation exist for treatment of these fractures. However, an optimal fixation technique has not yet been determined. Locking-plate technology has been applied to and popularized for the fixation of unstable PHFs. The locking-plate- screw construct acts as a fixed angle device to provide angular stability across the fracture, while the divergent configuration of the associated screws in the humeral head is theorized to improve a resistance to pullout. ${ }^{2-4)}$ Common complications of the locking-plate fixation method include screw perforation of the articular surface and varus collapse of the fracture; these types of complications occur most frequently after fixation of fractures in osteoporotic bone and fractures with medial metaphyseal comminution. ${ }^{2,5)}$

Restoration of the medial metaphysis is considered the most reliable predictor of a successful clinical outcome in unstable

Received September 27, 2018. Revised December 20, 2018. Accepted January 21, 2019.

Correspondence to: Yoon-Suk Hyun

Department of Orthopadic Surgery, Kangdong Sacred Heart Hospital, 150 Seongan-ro, Gangdong-gu, Seoul 05355, Korea

Tel: +82-2-2224-2230 1, Fax: +82-2-489-4391, E-mail: y1205s@hallym.or.kr, ORCID: https://orcid.org/0000-0003-3826-2527

IRB approval: Kangdong Sacred Heart Hospital (No. KANGDONG 2018-08-009-001).

Financial support: None. Conflict of interests: None. 
PHFs treated with locking-plate fixation. ${ }^{6,7)}$ For restoration of the medial metaphyseal area in PHFs in which the medial cortical support is broken and disrupted, fixation via a strut support with a fibular allograft and insertion of calcar screws has been used and that approach's efficacy has been evaluated in recent years. ${ }^{2,8-19)}$ We have had several bad experiences with the application of fibular allografts in unstable PHFs, including graft breakage during drilling, further displacement of the fracture gap for long graft insertion, and reduction loss with later graft displacement after graft fixation with push screws.

Impaction of tricortical bone blocks into a fracture's cavitary defect is familiar to many orthopedic surgeons and is relatively easy to perform. However, there are no previous reports on the use of tricortical bone block augmentation in the surgical treatment of unstable PHFs. Therefore, we decided to evaluate the results of PHF repair with the insertion of tricortical iliac allografts (TIAs) rather that fibular allografts as a method of augmenting of the medial column. The hypothesis in our study is that augmentation via TIAs in unstable PHFs can produce good clinical and radiological results regardless of the medial column support status after open reduction.

\section{Methods}

Institutional Review Board approval (No. KANGDONG 2018-08-009-001) was obtained for this investigation. We retrospectively evaluated 38 patients (14 males and 24 females) with unilateral displaced PHFs that were treated at our institute by a single surgeon between March 2015 and March 2017 using locking-plate fixation with TIA augmentation. The insertion of a TIA was indicated when an unstable PHF in preoperative images showed a large cavitary defect and poor medial column support after open reduction (regardless of the Neer classification), the existence of osteoporosis on preoperative evaluation of bone marrow density, and presence of medial cortical comminution in preoperative images. In unstable PHFs, initial collapse of fracture fragments resulting from high energy trauma, as well as from pushing on the head fracture fragment with a bone impactor during indirect open reduction, can create a large cavitary defect (Fig. 1). In some cases, augmentation with a strut graft for restoration of normal anatomy may not be required if the surgeon is able to obtain satisfactory medial column support and an acceptable humeral neck-shaft angle (HNSA) after open reduction using the intended impaction technique, even if medial metaphyseal comminution is present in preoperative images. In this study, we defined 'poor' medial column support as the absence
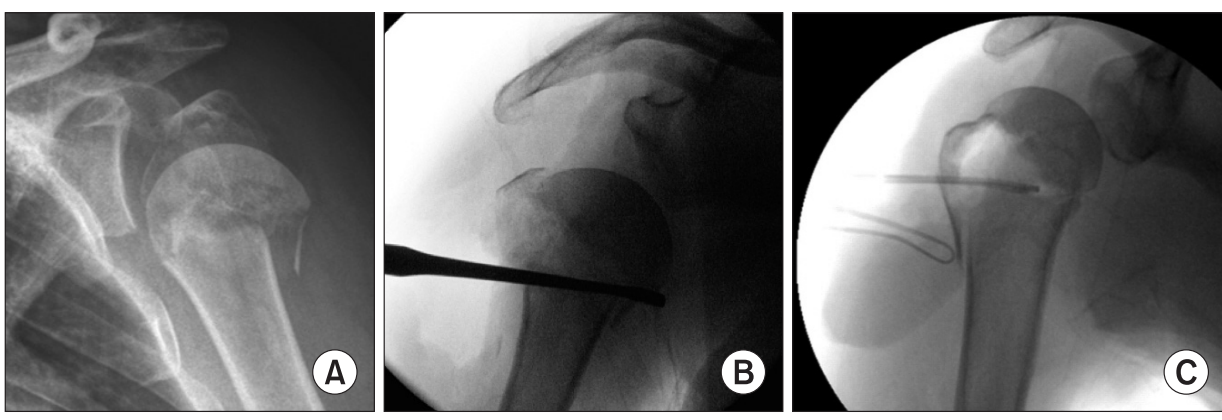

Fig. 1. Initial collapse of fracture fragments (A) by high energy trauma and subsequent pushing on the head fracture fragment with a bone impactor and periosteal elevator during indirect open reduction (B) can produce a large cavitary defect (C).
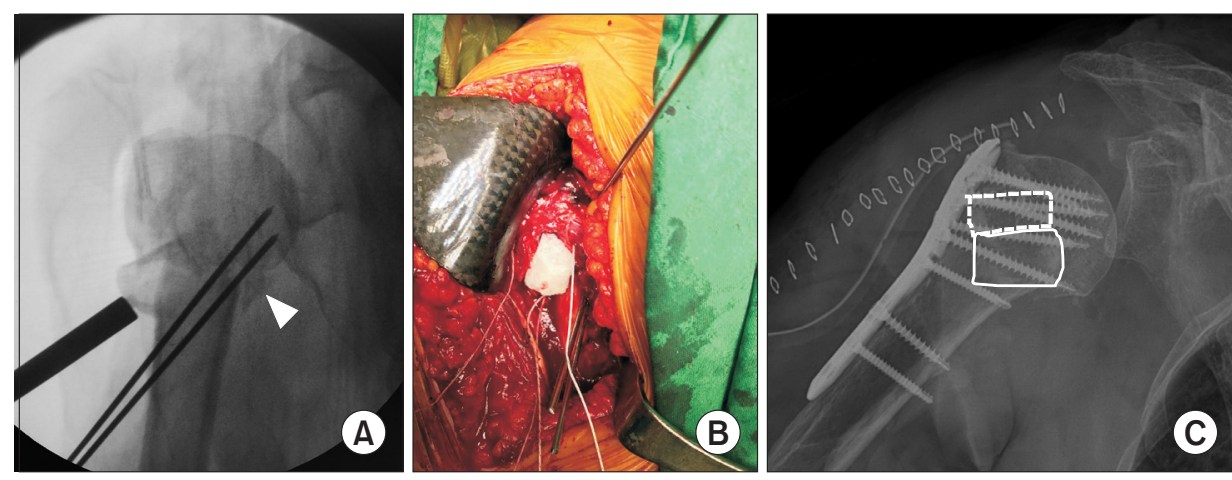

Fig. 2. (A) We defined 'poor medial column support' as the absence of definite medial cortical contact between the head and shaft fragments after open reduction under fluoroscopy (arrowhead). (B) A relatively low level of invasiveness during insertion of tricortical iliac allograft through the space between fracture fragments can maintain the established anatomical reduction under fluoroscopic surveillance. (C) When more than one bone block was used, the first bone block (solid line) was usually placed on the entry to the intramedullary space in the diaphysis. Additional bone blocks (dotted line) could be inserted to fill the remaining cavitary defect while maintaining the established anatomical reduction under fluoroscopic surveillance. 
of a definite medial cortical contact between humeral head and shaft fragments after open reduction under fluoroscopy (Fig. 2A). For each case, whether a large cavitary defect and poor medial column support were present was recorded during the perioperative recording session. We did not insert a TIA when the PHF did not have a large cavitary defect and had a definite medial cortical contact between humeral head and shaft fragments after open reduction.

The mean age of the patients at the time of surgery was 68.8 years (range, 49-88 years). The average follow-up duration was $15.6 \pm 4$ months. Among the 38 patients, 21 patients had both a large cavitary defect and poor medial column support after open reduction and were placed in group A. The remaining 17 patients had a large cavitary defect but no poor medial column support after open reduction and were placed in group B. Patient data were obtained from medical records and radiographs. Patients who failed to attend regularly scheduled follow-ups or did not complete their postoperative assessment were recalled for a complete evaluation.

Preoperatively, all patients underwent anteroposterior (AP) and lateral radiography of the shoulder, as well as computed tomography shoulder scans, on admission. Fractures were classified according to the Neer classification system after examining all preoperative imaging results. Among the PHFs, there were 11 two-part, 25 three-part, and two four-part fractures according to the Neer classification (Table 1).

All procedures were performed by a single surgeon (YSH). For surgery, patients were placed in a beach chair position under general anesthesia with the affected extremity free and draped. An image intensifier was included in the sterile surgical field. A standard deltopectoral approach was used. Open reduction

Table 1. Demographic Data

\begin{tabular}{|c|c|c|c|}
\hline \multirow{2}{*}{ Variable } & \multicolumn{2}{|c|}{$\begin{array}{l}\text { Status of medial column support } \\
\text { after open reduction }\end{array}$} & \multirow{2}{*}{$p$-value } \\
\hline & Poor & Good & \\
\hline No. of patient & 21 & 17 & \\
\hline Male:female & 7:14 & $7: 10$ & \\
\hline Age (yr) & $66.9(51-79)$ & $71.0(49-88)$ & 0.322 \\
\hline $\operatorname{BMD}\left(\mathrm{g} / \mathrm{cm}^{2}\right)$ & $-2.09 \pm 1.25$ & $-2.28 \pm 1.21$ & 0.663 \\
\hline \multicolumn{4}{|l|}{ Neer classification } \\
\hline 2 parts & 7 & 4 & \\
\hline 3 parts & 13 & 12 & \\
\hline 4 parts & 1 & 1 & \\
\hline Follow-up period (mo) & $15.5 \pm 4.3$ & $15.9 \pm 3.8$ & 0.750 \\
\hline
\end{tabular}

$\overline{\text { Values are presented as number only, median (range), or mean } \pm \text { standard }}$ deviation.

BMD: bone mineral density. was performed carefully so as not to create additional injury to soft tissues attached to fracture fragments. For displaced fracture fragments attached to a rotator cuff, non-resorbable FiberWire No. 5 (Arthrex, Naples, FL, USA) was placed transversely in the tendinous portion for later manual traction. Blunt instruments (periosteal elevator and bone impactor with $15 \mathrm{~mm}$ head diameter) were inserted through a space between the lateral fracture fragments (humeral shaft and tuberosities; Fig. 2C), and the upper part of the head fragment was gently pushed up while the lower part was pushed medially with distal traction of the humeral shaft by using a bone hook to attempt normal anatomy restoration, which was confirmed by fluoroscopy. The reduced fractures were fixed provisionally with two $2.0 \mathrm{~mm}$ diameter $\mathrm{K}$ wires (Fig. 2A, B). After obtaining anatomical reduction, if a large cavitary defect and poor medial cortical support were observed in fluoroscopic views, TIA bone blocks were inserted while maintaining the established anatomical reduction under fluoroscopic surveillance (Fig. 2A, B).

Insertion of a bone block larger than the defect could disrupt the established anatomical reduction during the insertion procedure or could impede anatomical reduction restoration of the displaced tubercular fracture fragments over the inserted allograft blocks, while a smaller bone block could escape through a medial fracture space or into the intramedullary space in the diaphysis. To avoid such issues, we estimated the size of the required bone block(s) based on intraoperative measurements obtained via probe and ruler. Because the sizes of commercially available TIAs vary, the TIAs used in this study were customized before insertion by using an electrical saw to ensure they appropriately fit the estimated size of the cavitary defect. We also recognized that insertion of additional bone blocks was an efficient method to fill the cavitary defect when remnant defect areas around the first inserted bone block were noticed, which occurred in many cases even though we had customized the bone block to the estimated defect size (Fig. 2C). When more than one bone block was used, the first bone block was usually placed on the entry of the intramedullary space in the diaphysis. Remnant bone blocks were then inserted to fill the remaining defect area while maintaining the established anatomical reduction under fluoroscopic surveillance. After confirmation of a satisfactory reduction of the displaced tubercular fracture fragment and application of manual traction via the nonabsorbable sutures, an anatomical locking compression plate, Humeral SuturePlate (Arthrex) was applied while maintaining manual traction of the sutures. Nonabsorbable sutures were then passed through the plate's suture holes and tied for maintenance of fracture reduction.

Our postoperative rehabilitation protocol consisted of supervised passive and active assisted ranges of motion therapies starting on postoperative day 2. Abduction and elevation up to $60^{\circ}$, without forced external rotation, were allowed for the first 8 weeks; thereafter, active exercises over the full range of motion 
were applied.

Patients were examined clinically and radiographically at 2, $6,12,24$, and 52 weeks postoperatively and on a yearly basis afterward. Postoperative clinical assessments included determination of Constant scores and shoulder range of motion. Passive shoulder range of motion parameters (forward flexion, abduction, external rotation at the side, and internal rotation at the back) were measured at the final follow-up visit by a single examiner using a goniometer. Radiographic views routinely obtained at all patient visits included shoulder true AP, scapula lateral $(\mathrm{Y})$, and axillary views. Maintenance of reduction was assessed via measurement of HNSA, humeral head height $(\mathrm{HHH})$, and head mediolateral offset (HMLO), as well, the status of the union was evaluated (Fig. 3). Regarding the angle between the humeral head and the shaft in the shoulder true AP view (i.e., $\mathrm{HNSA}$ ), restoration of the HNSA to $130^{\circ} \pm 10^{\circ}$ was considered good, an HNSA from $100^{\circ}$ to $120^{\circ}$ was considered fair, and an HNSA $<100^{\circ}$ was thought to be a poor outcome; based on the Paavolainen method. ${ }^{20)}$ The $\mathrm{HHH}$ was the distance between the upper end of the plate and the upper end of the humeral head and was used to evaluate the loss of reduction in shoulder true AP view (Fig. 3). ${ }^{9)}$ The HMLO was the distance from the lateral most portion of the greater tuberosity to the medial most portion of the humeral head (i.e., the distance between a straight

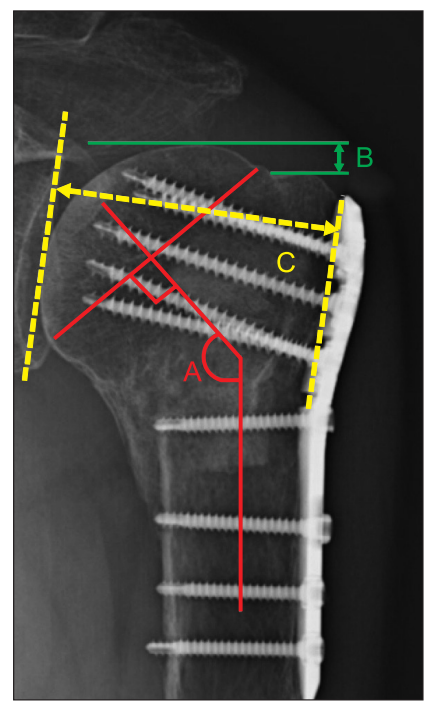

Fig. 3. Measurements used to determine humeral neck-shaft angle (HNSA), humeral head height $(\mathrm{HHH})$, and humeral mediolateral offset (HMLO). HNSA (A, red lines) was defined as the angle between a line along the humeral shaft axis and a line perpendicular to the anatomical neck; HHH (B, green lines) was defined as the vertical distance between a tangent line at the highest point of the humeral head and a similar line at the highest point of the greater tuberosity; HMLO (C, yellow lines) was defined as the distance from the lateral most portion of the greater tuberosity to the medial most portion of humeral head, which was obtained by drawing a straight line along the medial border of the plate and then drawing a parallel line at the medial most portion of humeral head in a shoulder true AP view and calculating the distance between the two lines. line drawn along the medial border of the plate and a parallel line drawn at the medial most portion of the humeral head in a shoulder true AP view; Fig. 3). Each measurement was obtained by using a picture archiving and communication system ( $\pi$ view; Mediface, Seoul, Korea). Loss of anatomic reduction was present if, in a comparison of immediate postoperative radiologic results with those at final post-surgical follow-up, the varus change of the HNSA was greater than $10^{\circ}$ or if the $\mathrm{HHH}$ change was more than $3 \mathrm{~mm}$.

Peri- and postoperative surgical complications, including screw penetration or cutout, humeral head avascular necrosis $(\mathrm{AVN})$, peri-implant fracture, infection, adhesive capsulitis, and need for further surgery were recorded. Intra-articular screw penetration was defined as screw violation of the articular surface without an associated loss of reduction, whereas screw cutout was considered present if a screw penetrated the articular surface and was associated with a loss of reduction.

We compared Constant scores, HNSA, range of shoulder motions at last evaluation, average period to bony union, and changes of $\mathrm{HHH}$ and $\mathrm{HMLO}$ offset during follow-up period in both patient groups. The Mann-Whitney U-test was used to compare the results of the two groups. Data analysis was performed using the IBM Statistical Package for the Social Sciences software ver. 20 (IBM Corp., Armonk, NY, USA). The statistical level of significance was set at $p<0.05$.

\section{Results}

Based on based on clinical and radiographical results, all fractures healed, and the average duration to complete union after surgery was 5.8 months. The patients' demographic data are summarized in Table 1. The average follow-up period was 13 months, and the mean Constant score was 73 points. The mean active forward flexion angle was $148^{\circ}$ while internal rotation, external rotation, and abduction were $38^{\circ}, 52^{\circ}$, and $122^{\circ}$, respectively. One case was determined to have a loss of anatomic reduction, exhibiting a $20^{\circ} \mathrm{HNSA}$ loss during follow-up (Fig. 4). Based on the Paavolainen method, 33 patients had good results and 5 patients had fair results at their final evaluation. With regard to $\mathrm{HHH}$ and $\mathrm{HMLO}$, the mean loss of reduction was 1.32 $\pm 0.65 \mathrm{~mm}$ in $\mathrm{HHH}$ and $5.02 \% \pm 3.1 \%$ in HMLO. Neither varus collapse with an $\mathrm{HNSA}$ of $<100^{\circ}$ nor AVN of the humeral head were detected during the follow-up period. None of the parameters evaluated showed a statistically significant difference between the two groups (Table 2).

\section{Discussion}

The purpose of this study was to analyze the clinical and radiographic outcomes of unstable PHFs treated by applying a locking plate, screws, and a TIA. The results showed that, 

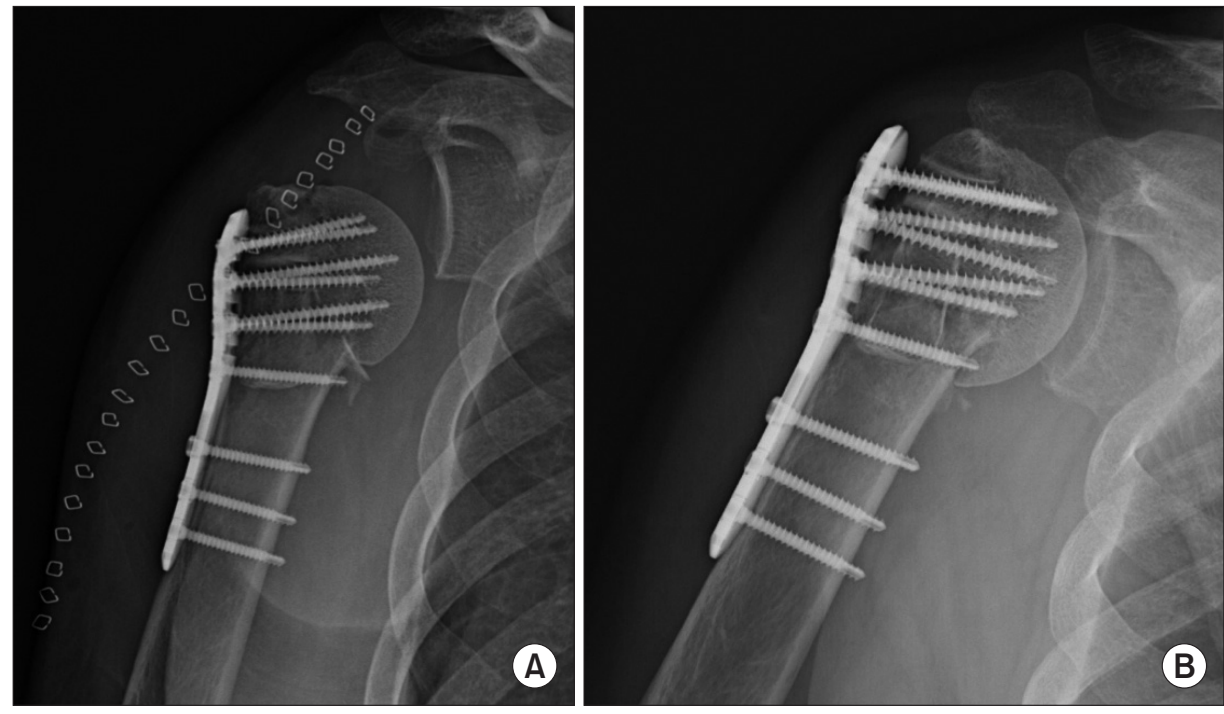

Fig. 4. A 71-year-old female included in our study showed loss of screw purchase in the distal area of the head fragment and little loss of reduction. Her humeral neck-shaft angle (HNSA) changed from $135^{\circ}$ to $105^{\circ}$ at final visit, but her final HNSA was deemed fair and adequate. (A) Simple radiograph at surgery day; (B) simple radiograph at final visit.

Table 2. Clinical and Radiologic Results ${ }^{\star}$

\begin{tabular}{|c|c|c|c|c|}
\hline \multirow{2}{*}{ Variable } & \multicolumn{3}{|c|}{ Status of medial column support after open reduction } & \multirow{2}{*}{$p$-value } \\
\hline & Total & Poor & Good & \\
\hline Constant score & $73 \pm 9.9$ & $70 \pm 9.9$ & $75 \pm 9.6$ & 0.121 \\
\hline \multicolumn{5}{|l|}{$\operatorname{ROM}\left({ }^{\circ}\right)$} \\
\hline $\mathrm{FF}$ & $148 \pm 16$ & $146 \pm 14$ & $150 \pm 17$ & 0.352 \\
\hline Abd & $122 \pm 17$ & $117 \pm 16$ & $130 \pm 17$ & 0.068 \\
\hline ER90 & $52 \pm 15$ & $53 \pm 11$ & $48 \pm 18$ & 0.399 \\
\hline IR90 & $38 \pm 17$ & $37 \pm 15$ & $39 \pm 20$ & 0.706 \\
\hline Change of $\mathrm{HHH}(\mathrm{mm})$ & $1.32 \pm 0.65$ & $1.29 \pm 0.63$ & $1.35 \pm 0.72$ & 0.561 \\
\hline Change of HMLO offset (\%) & $5.02 \pm 3.1$ & $4.95 \pm 2.9$ & $5.11 \pm 3.4$ & 0.861 \\
\hline Union & $5.8 \pm 2.8$ & $5.7 \pm 2.1$ & $6.0 \pm 2.6$ & 0.716 \\
\hline \multicolumn{5}{|l|}{$\mathrm{NSA}^{\dagger}$} \\
\hline Good & 33 & 18 & 15 & \\
\hline Fair & 5 & 3 & 2 & \\
\hline
\end{tabular}

Values are presented as mean \pm standard deviation or number only.

ROM: range of motion, FF: forward flexion, Abd: abduction, ER: external rotation, IR: internal rotation, HHH: humeral head height, HMLO: head mediolateral offset, NSA: neck-shaft angle.

*Values in Grade of NSA, Constant scores, and each motion range were at last follow-up.

${ }^{\dagger}$ Based on the Paavolainen method.

regardless of the condition of the medial column support after open reduction (poor or not poor), fixation with TIA augmentation showed good radiological and clinical results. There were no significant differences detected among any of the assessed parameters between patients with and without poor medial column support at final postoperative visit. These results support our hypothesis that TIA augmentation in unstable PHFs can produce good clinical and radiological results, regardless of medial column support status after open reduction.

Impaired bone quality and considerable comorbidities pose special challenges in treating PHFs in an aged population with locking-plate fixation systems. Locking-plate fixation has become a standard treatment in stabilizing PHFs, but it is associated with a surgical revision rate of up to $25 \%$, a rate that seems to have stagnated at this relatively high level. ${ }^{21-24)}$ Complications after operative treatment are frequent, in particular, those associated with loss of reduction with varus malalignment and subsequent screw cutout.

Several previous studies have shown that medial osseous stability of the humerus is an essential prerequisite for a satisfactory 
functional outcome of patients with unstable PHFs. ${ }^{6-9,25)}$ The loss of medial support is the most common reason for secondary displacement with varus impaction. ${ }^{9,25)} \mathrm{A}$ varus displacement of $20^{\circ}$ significantly increases rotator cuff forces in elevatory movements, which can severely limit functional outcome. ${ }^{25)}$ Gardner et al. ${ }^{9}$ showed that the loss of medial support results in a five-fold higher locking screw cutout rate. In their analysis, locking-plate fixation was considered to provide adequate medial support if either the medial cortex is intact, anatomically reduced, and not comminuted or if there is stable head-on-shaft impaction or if a superiorly directed oblique locking screw is appropriately placed into the inferomedial quadrant of the proximal humeral head fragment.

Restoration of medial column support with a locking plate, calcar screw insertion, and fibular strut graft augmentation has been a representative restorative tool for PHFs, and there is abundant evidence of their use and effectiveness. ${ }^{2,8-18)}$ However, in this study, we chose to use a TIA instead of a fibular strut allograft.

\section{Why the Authors Chose Tricortical Iliac Allografts and the Advantages of That Selection}

Based on our experiences in previous cases, fracture fragments can be displaced further during the insertion of fubular allograft and straining during the surgical procedure to insert a long fibular strut into the gap between each fracture fragment can result in additional injury to the intact soft tissue bridge between the humeral head and shaft fragments. Moreover, a mismatch between a customized fibula insert and the actual cavitary defect can prolong the duration of the surgery. In contrast, insertion of tricortical bone blocks does not require additional fixation, such as the 'push screw' required for fibular strut graft insertion, to reduce the medial column through the apposition of fibular graft. By gradually packing tricortical bone blocks under fluoroscopic guidance, we were able to obtain adequate restoration of normal anatomy with no need for additional fixation of the allograft. Finally, compared to fibular strut allografts, there is less need for customized cutting of tricortical bone blocks to fit into the fracture's cavitary defect.

\section{Authors' Indication of Using Bone Block Augmentation and Its Background}

Due to the presence of neighboring neurovascular structures and the nearby areas of insertion of the rotator cuff and biceps tendons, extramedullary fixation of PHFs is most commonly approached from a lateral aspect. ${ }^{26,27}$ On that basis, reduction of a medial fracture zone can only be achieved through indirect manipulation. Fractures with medial comminution are technically difficult or not at all manageable because direct visual control is impossible, and the treatment of choice in such cases has been the intended impaction of the humeral head. ${ }^{21)}$ The presence of medial comminution of metaphyseal and cortical areas does not always mean there is poor medial column support, although severe comminution in medial metaphyseal and cortical areas can result in poor medial column support. Based on author's experiences, even if medial metaphyseal comminution was observed in preoperative images, augmentation with a strut graft for restoration of normal anatomy may not be required because we have been able to attain satisfactory medial column support with an acceptable HNSA after open reduction with the locking-plate and TIA argumentation approach (Fig. 1C). In other words, if the level of medial metaphyseal comminution is not severe, we can achieve satisfactory medial column support after open reduction and good clinical and radiologic results without using strut graft augmentation.

We have defined 'poor' medial column support as the absence of a definite medial cortical contact between head and shaft fragments after open reduction under fluoroscopy. In open reduction with bone impactors and fibular strut allograft augmentation cases, we had several bad outcomes including varus collapse and/or screw cutout, and poor clinical outcomes could occur when there were a large cavitary defect and a thin head fragment (too thin for firm inferomedial screw fixation) present. Based on those experiences, we developed our own indicators for augmentation via strut allograft: i) presence of an unstable PHF that in preoperative images has a large cavitary defect and poor medial column support after open reduction, regardless of the Neer classification; ii) existence of osteoporosis upon preoperative evaluation of bone marrow density; and iii) severe medial cortical comminution in preoperative images. We do not decide on the insertion of bone blocks preoperatively because, based on our experiences, medial comminution and poor medial column support in preoperative images may indicate a case that did not need augmentation after open reduction. Therefore, the decision to insert bone blocks is made after open reduction and is indicated when there is poor medial column support, a large cavitary defect, and a thin humeral head fragment. In our experience, thin subchondral bone in the head fracture fragment is usually accompanied by a large cavitary defect after open reduction with a bone impactor, making it made difficult to obtain firm screw purchase in the inferior part of humeral head fragment. In such cases, poor purchase of an inferomedial screw into the humeral head fragment and the presence of a thin fragment can result in screw cutout with varus collapse, screw pullout, and other complications. We believe that, at a minimum, TIA bone blocks can help to prevent screw cutout and varus collapse when thin subchondral bone in the humeral head fracture fragment is accompanied with a large cavitary defect. Bone block insertion is not used when medial cortical contact is sufficient and there is an indication of firm purchase of inferomedial screws, even when there are small to medium sized cavitary defects after open reduction. Regardless, we recognize that a large 
cavitary defect between fracture fragments usually occurs when the humeral head fragment is severely collapsed and thin due to the fracture event, the reduction procedure, or when the medial cortical contact could not recreate a normal neck-shaft angle.

In many PHF cases, preoperative plans regarding the insertion of bone blocks were changed after the open reduction procedure. In this study, one case (71-year-old female, 4 part fracture) showed a loss of screw purchase in the distal area of the head fragment along with some loss of reduction. Her HNSA changed from $135^{\circ}$ to $105^{\circ}$ but her final HNSA was deemed fair and adequate (Fig. 4). Regarding screw purchase in the head fragment of this case, bone block augmentation alone could not provide solid fixation on the head fragment; if there was insufficient insertion of each screw in the head fragment, screws could pull out and the head fragment could be displaced.

\section{Head Mediolateral Offset}

A review article by Saltzman et al. ${ }^{28)}$ reported that PHFs with preoperative displacement, varus coronal malalignment, and/or medial cortical comminution are not indications for fibular strut allograft augmentation due to the increased risk of screw pullout, varus malreduction, and humeral head subsidence, resulting in poor functional outcomes. Increased tuberosity lateralization is important for restoring rotator cuff lever arm in elevation and rotation, ${ }^{29)}$ and failure to restore humeral offset, defined as the distance between the center of rotation and the lateral cortex of the greater tuberosity, is associated with poor functional outcomes in acute PHFs. ${ }^{30)}$ In addition, HMLO may be related to varus deformation of the humeral head and loss of normal range of HNSA. Thus, we examined HMLO during our radiological evaluation of outcome; however, there are no previous reports on HMLO and osteosynthesis in PHFs. In our cases, regardless of patient group, HMLO levels were relatively consistent (range, $0 \%-10 \%)$; however, whether the values can be deemed to be good or bad was not evaluated formally. Regardless, we consider HMLO measurement valuable when evaluating a clinical outcome after osteosynthesis with locking-plate fixation of unstable PHFs.

The retrospective nature of our study is a weak point. However, all cases treated with TIA within the study period were included in our study, and there was no loss of follow-up. In addition, all cases were treated by the same surgeon, so bias related to differences in surgeon skill can be ignored. A control group was not included in this study. In a future study, a control group comprised of patients with poor medial column support without graft augmentation may be the best option to elucidate the efficacy of TIA insertion in unstable PHFs. Poor medial column support in unstable PHFs is considered a powerful risk factor that can affect clinical and radiological outcomes. The lack of significant differences between the two groups in this study and the relatively good outcomes in the patients in our study support our conclusion that locking-plate fixation with TIA augmentation can useful in treating unstable PHFs, including those with poor medial column support.

\section{Conclusion}

In patients with unstable PHFs, augmentation using TIA and fixation with a locking plate provided good clinical and radiological results, even when there was poor support of the medial column and a large cavitary defect after open reduction.

\section{References}

1. Connor PM, Flatow EL. Complications of internal fixation of proximal humeral fractures. Instr Course Lect. 1997;46:25-37.

2. Chow RM, Begum F, Beaupre LA, Carey JP, Adeeb S, Bouliane MJ. Proximal humeral fracture fixation: locking plate construct \pm intramedullary fibular allograft. J Shoulder Elbow Surg. 2012;21(7):894-901. doi: 10.1016/j.jse.2011.04.015.

3. Frigg R. Locking Compression Plate (LCP). An osteosynthesis plate based on the Dynamic Compression Plate and the Point Contact Fixator (PC-Fix). Injury. 2001;32 Suppl 2:63-6. doi: 10.1016/S0020-1383(01)00127-9

4. Frigg R. Development of the locking compression plate. Injury. 2003;34 Suppl 2:B6-10. doi: 10.1016/j.injury.2003.09.020.

5. Haasters F, Siebenbürger G, Helfen T, Daferner M, Böcker W, Ockert B. Complications of locked plating for proximal humeral fractures-are we getting any better? J Shoulder Elbow Surg. 2016;25:e295-303. doi: 10.1016/j.jse.2016.02.015.

6. Lee CW, Shin SJ. Prognostic factors for unstable proximal humeral fractures treated with locking-plate fixation. J Shoulder Elbow Surg. 2009;18(1):83-8. doi: 10.1016/j.jse.2008.06.014.

7. Jung WB, Moon ES, Kim SK, Kovacevic D, Kim MS. Does medial support decrease major complications of unstable proximal humerus fractures treated with locking plate? BMC Musculoskelet Disord. 2013;14:102. doi: 10.1186/1471-2474-14102.

8. Ponce BA, Thompson KJ, Raghava P, et al. The role of medial comminution and calcar restoration in varus collapse of proximal humeral fractures treated with locking plates. J Bone Joint Surg Am. 2013;95(16):e113(1-7). doi: 10.2106/JBJS.K.00202.

9. Gardner MJ, Weil Y, Barker JU, Kelly BT, Helfet DL, Lorich DG. The importance of medial support in locked plating of proximal humerus fractures. J Orthop Trauma. 2007;21(3):185-91. doi: 10.1097/BOT.0b013e3180333094.

10. Schliemann B, Wähnert D, Theisen C, et al. How to enhance the stability of locking plate fixation of proximal humerus fractures? An overview of current biomechanical and clinical data. Injury. 2015;46(7):1207-14. doi: 10.1016/j.injury.2015. 04.020 .

11. Gardner MJ, Boraiah S, Helfet DL, Lorich DG. Indirect medial 
reduction and strut support of proximal humerus fractures using an endosteal implant. J Orthop Trauma. 2008;22(3):195200. doi: 10.1097/BOT.0b013e31815b3922.

12. Bae JH, Oh JK, Chon CS, Oh CW, Hwang JH, Yoon YC. The biomechanical performance of locking plate fixation with intramedullary fibular strut graft augmentation in the treatment of unstable fractures of the proximal humerus. J Bone Joint Surg Br. 2011;93(7):937-41. doi: 10.1302/0301-620X.93B7.26125.

13. Chen H, Ji X, Zhang Q, Liang X, Tang P. Clinical outcomes of allograft with locking compression plates for elderly four-part proximal humerus fractures. J Orthop Surg Res. 2015;10:114. doi: 10.1186/s13018-015-0258-9.

14. Mathison C, Chaudhary R, Beaupre L, Reynolds M, Adeeb S, Bouliane M. Biomechanical analysis of proximal humeral fixation using locking plate fixation with an intramedullary fibular allograft. Clin Biomech (Bristol, Avon). 2010;25(7):642-6. doi: 10.1016/j.clinbiomech.2010.04.006.

15. Kim DS, Lee DH, Chun YM, Shin SJ. Which additional augmented fixation procedure decreases surgical failure after proximal humeral fracture with medial comminution: fibular allograft or inferomedial screws? J Shoulder Elbow Surg. 2018;27(10):1852-8. doi: 10.1016/j.jse.2018.03.020.

16. Cha H, Park KB, Oh S, Jeong J. Treatment of comminuted proximal humeral fractures using locking plate with strut allograft. J Shoulder Elbow Surg. 2017;26(5):781-5. doi: 10.1016/j.jse.2016.09.055.

17. Panchal K, Jeong JJ, Park SE, et al. Clinical and radiological outcomes of unstable proximal humeral fractures treated with a locking plate and fibular strut allograft. Int Orthop. 2016;40(3):569-77. doi: 10.1007/s00264-015-2950-0.

18. Newman JM, Kahn M, Gruson KI. Reducing postoperative fracture displacement after locked plating of proximal humerus fractures: current concepts. Am J Orthop (Belle Mead NJ). 2015;44(7):312-20.

19. Berkes MB, Little MT, Lazaro LE, et al. Intramedullary allograft fibula as a reduction and fixation tool for treatment of complex proximal humerus fractures with diaphyseal extension. J Orthop Trauma. 2014;28(3):e56-64. doi: 10.1097/ BOT.0b013e31829a346d.

20. Paavolainen P, Björkenheim JM, Slätis P, Paukku P. Operative treatment of severe proximal humeral fractures. Acta Orthop
Scand. 1983;54(3):374-9.

21. Laux CJ, Grubhofer F, Werner CML, Simmen HP, Osterhoff G. Current concepts in locking plate fixation of proximal humerus fractures. J Orthop Surg Res 2017;12(1):137. doi: 10.1186/ s13018-017-0639-3.

22. Schliemann B, Siemoneit J, Theisen Ch, Kösters C, Weimann A, Raschke MJ. Complex fractures of the proximal humerus in the elderly--outcome and complications after locking plate fixation. Musculoskelet Surg. 2012;96 Suppl 1:S3-11. doi: 10.1007/s12306-012-0181-8.

23. McMillan TE, Johnstone AJ. Primary screw perforation or subsequent screw cut-out following proximal humerus fracture fixation using locking plates: a review of causative factors and proposed solutions. Int Orthop. 2018;42(8):1935-42. doi: 10.1007/s00264-017-3652-6.

24. Sproul RC, lyengar JJ, Devcic Z, Feeley BT. A systematic review of locking plate fixation of proximal humerus fractures. Injury. 2011;42(4):408-13. doi: 10.1016/j.injury.2010.11.058.

25. Brunner F, Sommer C, Bahrs C, et al. Open reduction and internal fixation of proximal humerus fractures using a proximal humeral locked plate: a prospective multicenter analysis. J Orthop Trauma. 2009;23(3):163-72. doi: 10.1097/BOT. 0b013e3181920e5b.

26. Gerber C, Schneeberger AG, Vinh TS. The arterial vascularization of the humeral head. An anatomical study. J Bone Joint Surg Am. 1990;72(10):1486-94.

27. Brooks $\mathrm{CH}$, Revell WJ, Heatley FW. Vascularity of the humeral head after proximal humeral fractures. An anatomical cadaver study. J Bone Joint Surg Br. 1993;75(1):132-6.

28. Saltzman BM, Erickson BJ, Harris JD, Gupta AK, Mighell M, Romeo AA. Fibular strut graft augmentation for open reduction and internal fixation of proximal humerus fractures: a systematic review and the authors' preferred surgical technique. Orthop J Sports Med. 2016;4(7):2325967116656829. doi: $10.1177 / 2325967116656829$.

29. Sirveaux F, Roche O, Molé D. Shoulder arthroplasty for acute proximal humerus fracture. Orthop Traumatol Surg Res. 2010; 96(6):683-94. doi: 10.1016/j.otsr.2010.07.001.

30. Demirhan M, Kilicoglu O, Altinel L, Eralp L, Akalin Y. Prognostic factors in prosthetic replacement for acute proximal humerus fractures. J Orthop Trauma. 2003;17(3):181-8; discussion 188-9. 